\title{
The reactivity to CK5/6 antibody in tumor cells from non-small cell lung cancers shed into pleural effusions predicts survival
}

\author{
JANNA SAND DEJMEK ${ }^{1}$ and ANNIKA DEJMEK ${ }^{2}$ \\ ${ }^{1}$ Department of Surgery, Malmö University Hospital; ${ }^{2}$ Department of Laboratory Medicine, \\ Lund University, Malmö University Hospital, Malmö, Sweden
}

Received August 11, 2005; Accepted October 13, 2005

\begin{abstract}
Lung cancer, especially adenocarcinoma and large cell carcinoma, tends to spread to the pleural cavities. Once an effusion develops, the prognosis is generally dismal. Immunocytochemistry is frequently applied to effusions for diagnostic purposes, but the prognostic value of markers in malignant effusions have thus far attracted less attention. Dakopatts CK 5/6 antibody was applied to ethanol-fixed fresh cytospin preparations from malignant pleural effusions originating from 18 patients (11 men and 7 women) with a previously or later verified non-small cell lung carcinoma (NSCLC). In three cases, CK5/6 reactivity was found in part of the malignant population, whereas 10 cases showed reactivity in most tumor cells. The lack of reactivity in malignant cells was only seen in five effusions. Females showed significantly lower reactivity rates, with all negative effusions coming from female patients, whereas 9/10 effusions with reactivity in most malignant cells originated from males. CK5/6 reactivity was significantly correlated to survival, with a median survival time of 18 days for patients with CK5/6-negative tumors, and 212 days for those with positive tumors. The strong relationship between CK5/6 reactivity and survival, and the observed gender difference, warrants larger studies aimed at the clinical utility of CK5/6 as a prognostic marker in metastatic NSCLC, the possible functional role of CK5/6 in cell adhesion in advanced NSCLC and its possible hormonal control.
\end{abstract}

\section{Introduction}

The epidemiology of lung cancer has changed during recent years. Reflecting changing smoking habits during the last decades, the incidence among women is still increasing, whereas the incidence among men is declining $(1,2)$.

Correspondence to: Dr Annika Dejmek, Department of Laboratory Medicine, Lund University, Malmö University Hospital, S-205 02 Malmö, Sweden

E-mail: annika.dejmek@pat.mas.lu.se

Key words: non-small cell lung carcinoma, CK5/6, pleural effusion, immunocytochemistry, survival, gender
Furthermore, the histological spectrum of non-small cell lung carcinomas (NSCLC) has changed in both sexes. An overall increase in adenocarcinomas has been seen, whereas squamous carcinomas are decreasing. The increase in adenocarcinomas is seen especially in women.

Adenocarcinomas and large cell carcinomas tend to grow in the periphery of the lungs and frequently spread to the pleural cavity, giving rise to a pleural effusion. Once an effusion has developed, the prognosis is dismal and median survival is comparable to stage IV disease $(3,4)$. However, survival times differ in advanced tumor stages, but predictors of prognosis for patients with malignant pleural effusions have thus far not been studied.

Thanks to the relatively selective expression of CK5 in mesothelial cells compared to adenocarcinoma, CK5/6 was originally proposed as a marker in the differentiation between mesothelioma and lung adenocarcinoma in histological material. Primary lung adenocarcinomas usually do not express CK5/6, whereas squamous carcinomas and many large cell carcinomas are CK5/6-positive. (5-9). CK5/6 reactivity has also been found in adenocarcinomas from various primary sites (10).

Immunocytochemistry is a widely used tool to characterize malignant cell populations in effusions (11-13). Adenocarcinomas and large cell carcinomas of the lung both tend to spread to the pleural cavities and cannot always be distinguished morphologically when they appear in effusions.

CK5/6 has so far not been tested on cytospin preparations from serous effusions. When applying CK5/6 to a series of effusions, we noticed a higher frequency of positive NSCLC than expected. Based on preliminary observations indicating gender differences, we applied CK5/6 to 18 malignant pleural effusions originating from 11 men and 7 women with NSCLC and analyzed the results with special reference to gender difference. Since differences in outcome between male and female lung cancer patient have been described, we also analyzed the value of CK5/6 expression as a prognostic marker.

\section{Materials and methods}

The study comprises malignant pleural effusions from 18 patients (11 men and 7 women) with NSCLC investigated at 
Table I. Antibodies used.

\begin{tabular}{lcc}
\hline Antibody & Catalog no. & Dilution \\
\hline CEA & M7072 & $1: 30$ \\
EMA & M0613 & $1: 50$ \\
BerEp4 & M804 & $1: 300$ \\
Vimentin & M0725 & $1: 250$ \\
Thrombomodulin & M617 & $1: 800$ \\
Ca125 & M3519 & $1: 400$ \\
HBME-1 & M3505 & $1: 50$ \\
Sialyl-Tn & M899 & $1: 400$ \\
CK5/6 & M7237 & $1: 10$ \\
CK7 & M7018 & $1: 100$ \\
CK20 & M7019 & $1: 200$ \\
TTF-1 & M3575 & $1: 10$ \\
\hline
\end{tabular}

the Department of Clinical Pathology and Cytology, Malmö University Hospital, from 2001 to 2002. All effusions containing malignant cells where a NSCLC was previously or later confirmed are included. Since the inclusion criterion is the occurrence of a malignant pleural effusion, the stage for all patients was consistent with IIIb or IV.

The final diagnoses were based on all available information, including biopsies, autopsies (if performed), fine needle aspiration biopsies from primary tumors and metastases, bronchial brushings, radiological findings, clinical information, electron microscopy (in one case), and follow-up.

For all fluids, hematoxylin-eosin- and Giemsa-stained smears were processed for morphological evaluation. All fluids were also immunostained with our standard panel for distinguishing between mesothelioma and adenocarcinoma in effusions (12).

The immunocytochemical stains were performed on cytospin preparations from fresh fluids. The slides were immediately fixed in $95 \%$ ethanol, and all immunostaining was performed with Dako Tech-Mate ${ }^{\mathrm{TM}} 500$ Plus (Dakopatts

Table II. Survival, diagnosis and reactivity in 18 cases.

\begin{tabular}{|c|c|c|c|c|c|c|c|c|c|c|c|c|c|c|c|}
\hline Gender & Age & $\begin{array}{c}\text { Survival } \\
\text { (days) }\end{array}$ & $\mathrm{CD}$ & $\begin{array}{c}\text { Final } \\
\text { diagnosis }\end{array}$ & CK5/6 & CEA & EMA & BerEp4 & Thromb & CA125 & Sialyl & HBME & CK 7 & CK20 & TTF-1 \\
\hline $\mathrm{F}$ & 76 & 8 & ACA & ACA & 0 & 0 & 2 & 2 & 2 & 2 & 0 & 0 & 2 & 0 & ND \\
\hline $\mathrm{F}$ & 64 & 18 & $\begin{array}{c}\text { CA, } \\
\text { poorly } \\
\text { diff }\end{array}$ & NSCLC & 0 & 0 & 2 & 1 & 1 & 2 & 0 & 0 & 2 & 0 & ND \\
\hline $\mathrm{F}$ & 65 & 250 & ACA & ACA & 0 & 1 & 2 & 2 & 1 & 0 & 1 & ND & 2 & 0 & 1 \\
\hline $\mathrm{F}$ & 80 & 10 & ACA & ACA & 0 & 1 & 2 & 2 & 1 & 0 & 1 & ND & 2 & 0 & 0 \\
\hline $\mathrm{F}$ & 78 & 22 & $\mathrm{ACA}$ & ACA & 0 & 1 & 2 & 2 & 0 & 0 & 2 & 0 & 2 & 0 & 2 \\
\hline $\mathrm{F}$ & 49 & 460 & ACA & ACA & 1 & 0 & 2 & 2 & 1 & 2 & 2 & 2 & 2 & 0 & 2 \\
\hline $\mathrm{F}$ & 67 & 263 & ACA & NSCLC & 2 & 1 & 2 & 2 & 1 & 2 & 1 & 1 & 2 & 1 & ND \\
\hline M & 70 & 44 & ACA & ACA & 1 & 0 & 2 & 2 & 0 & 2 & 3 & 0 & 2 & 0 & ND \\
\hline M & 69 & 443 & ACA & ACA & 1 & 1 & 2 & 2 & 1 & 1 & 1 & 0 & 2 & 0 & 2 \\
\hline M & 79 & 68 & $\begin{array}{c}\text { ACA, } \\
\text { poorly } \\
\text { diff }\end{array}$ & NSCLC & 1 & 0 & 2 & 2 & 1 & 2 & 0 & 2 & 2 & 0 & ND \\
\hline M & 55 & 51 & ACA & ACA & 2 & 2 & 2 & 2 & 2 & 2 & 2 & 0 & 2 & 0 & ND \\
\hline M & 58 & 48 & $\begin{array}{l}\text { Malig, } \\
\text { MM? }\end{array}$ & $\mathrm{ACA}^{\mathrm{a}}$ & 2 & 1 & 2 & 2 & 2 & 1 & 2 & 0 & 2 & 1 & ND \\
\hline M & 53 & 512 & ACA & ACA & 2 & 2 & 2 & 2 & 1 & 2 & 1 & 1 & 2 & 0 & ND \\
\hline M & 67 & 212 & ACA & ACA & 2 & 1 & 2 & 2 & 1 & 2 & 2 & 0 & 2 & 1 & ND \\
\hline M & 48 & 179 & ACA & ACA & 2 & 2 & 2 & 2 & 2 & 2 & 1 & 0 & 2 & 0 & ND \\
\hline M & 71 & 57 & ACA & ACA & 2 & 0 & 2 & 2 & 2 & 2 & 1 & 1 & 2 & 1 & ND \\
\hline M & 76 & 388 & $\begin{array}{c}\text { Malig, } \\
\text { poorly } \\
\text { diff, } \\
\text { MM/ } \\
\text { ACA? }\end{array}$ & NSCLC & 2 & 2 & 2 & 2 & 0 & 2 & 2 & 0 & ND & ND & ND \\
\hline M & 74 & 317 & ACA & ACA & 2 & 2 & 2 & 2 & 0 & 2 & 0 & 0 & 2 & 0 & ND \\
\hline
\end{tabular}



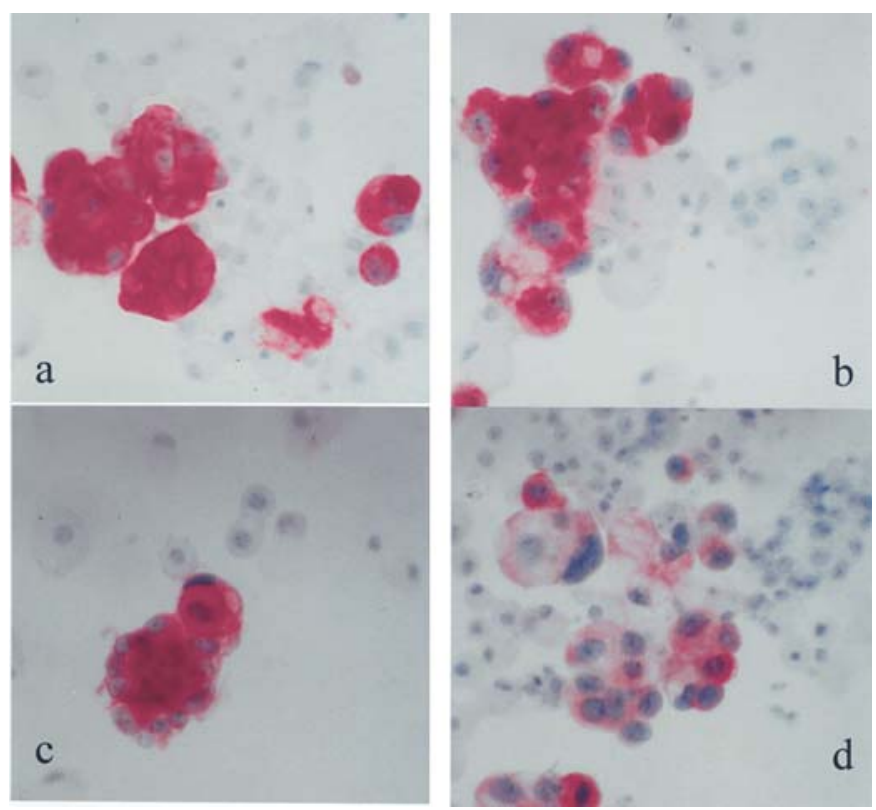

Figure 1. Cells from lung adenocarcinoma in pleural effusion from a 74-year old male, with a survival time of 317 days. (A) Strong reactivity to CK5/6 in the tumor and background mesothelial cells, negative macrophages. (B) Strong cytoplasmic EMA reactivity in tumor cells, negative mesothelial cells. (C) Strong BerEp4 reactivity in tumor cells, negative mesothelial cells . (D) Moderate CEA reactivity in tumor cells, negative mesothelial cells.
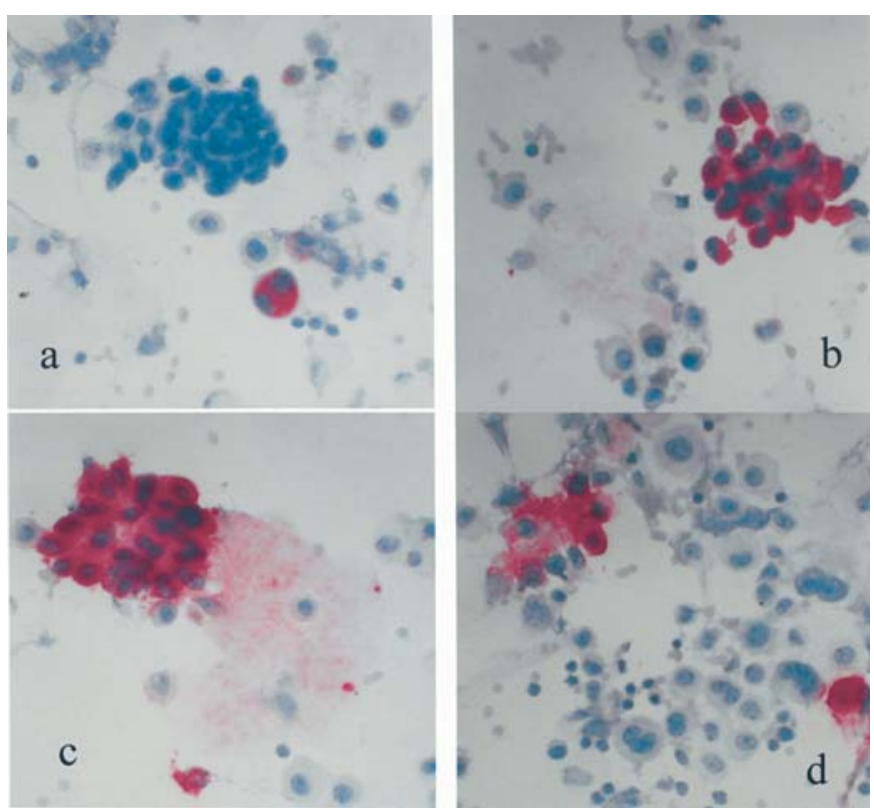

Figure 2. Cells from lung adenocarcinoma in pleural effusion from a 78-year old female, survival time 22 days. (A) Cluster of CK5/6-negative tumor cells, scattered mesothelial cells with strong or moderate reactivity, negative macrophages. (B) Strong cytoplasmic EMA reactivity in tumor cells, and no reactivity in mesothelial cells. (C) Strong BerEp4 reactivity in tumor cells, negative mesothelial cells. (D) Strong CEA reactivity in part of tumor cell population, negative mesothelial cells.

AB, Alvsjö, Sweden) using the alkaline phosphatase-antialkaline (APAAP) method (ChemMate ${ }^{\mathrm{TM}}$ detection kit no. K5000; Dako Cytomation) to visualize the antigen-antibody complex. The CK5/6 antibody from Dakopatts (no. 7237) was used. All antibodies used in this study are listed in Table I.

The intensity of the immunostaining was estimated, and the proportion of stained cells was evaluated semiquantitatively.
The reactivity was assigned to three groups: i) 2 , moderate or strong reactivity seen in most tumor cells ( $>70 \%$ of identifiable malignant cells); ii) 1 , moderate or strong reactivity in part of the tumor cell population $(>5-10 \%$ but $<70 \%$ of the malignant population); iii) 0 , no reactivity, weak reactivity or reactivity in $<5-10 \%$ of tumor cells. Moderate or strong reactivity found in occasional cells and all weak reactivity was considered negative (0). For EMA, membrane or cytoplasmic reactivity was recorded as a separate parameter. Staining in areas with degenerated cells and a proteinous background was not considered in the evaluation.

For all cases a negative control was included. A Giemsastained cytospin preparation was always included to facilitate the identification of relevant cells. Survival time was calculated in days from the first appearance of the effusion.

Statistical analysis. The results were evaluated using the Minitab 12 statistical package (Minitab Inc., State College, PA, USA), survival using Kaplan-Meier analysis with Wilcoxon's rank test, and differences in immunoreactivity using the Mann-Whitney median test.

\section{Results}

The results for each case are given in Table II. The total material falls into the range of other reported studies with respect to age (median, 68 years; mean $\pm \mathrm{SD}, 67 \pm 10$ ) and survival (median 124 days; mean \pm SD, 177 \pm 175 ).

The median age among the female patients was 67 years, with a mean \pm SD of $68 \pm 11$, and 69 years among the male patients with a mean \pm SD of $65 \pm 10$. Mean survival time in days for all female patients was 147 ( \pm SD 179) and 210 $( \pm$ SD 175$)$ for the male patients. The differences between men and women in age and Kaplan-Meier survival curves were not significant.

Positive cells showed distinct cytoplasmic staining. Macrophages often showed unspecific staining that was weak and uniform, were present in the entire macrophage population, and offered no interpretation problems.

Based on morphological features, the tumors were cytologically diagnosed as poorly differentiated carcinoma, adenocarcinoma, or poorly differentiated malignant tumor. There was no correlation between morphological features and differentiation and CK5/6 reactivity.

The observed CK 5/6 reactivity in tumor cells differed significantly $(\mathrm{p}=0.004)$ between male and female patients (Figs. 1 and 2). For CK5/6, all effusions from male patients were positive and $8 / 11$ cases had strong or moderate reactivity in the whole population of tumor cells. No females had effusions with strongly positive tumor cells and 2/7 effusions from female patients showed moderate reactivity in part of and most of the malignant cells, respectively. Because of the noted difference for CK5/6, we applied the same statistical analysis to the results of our routine panel, but no gender difference was found.

In combined results for both genders, positivity for CK5/6 was associated with vastly longer survival. Patients with CK5/6-positive tumors had a median survival time of 212 days vs. 18 days for those with CK5/6-negative tumors. The Kaplan-Maier survival curves were significantly different $(\mathrm{p}=0.0015)$ (Fig. 3). 


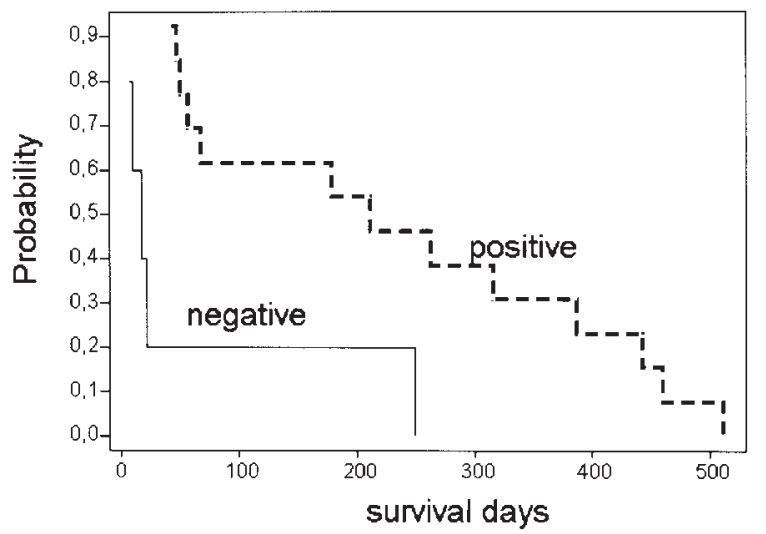

Figure 3. Survival times in days for patients with CK5/6-positive and -negative NSCLC tumor cells in effusions.

\section{Discussion}

We have studied the CK5/6 reactivity in NSCLC cells shed into effusions in patients with disseminated lung cancer. The results show a significant difference between men and women in the reactivity to the CK5/6 antibody in NSCLC cells in pleural effusions. The reactivity was not correlated to any differences in tumor morphology or differentiation seen in the effusions.

In those patients whose tumor cells in effusions were CK5/6 reactive, survival time was longer in magnitude, which is of importance for clinical management. The reported findings are based on a small series of samples, but are highly significant in the statistical sense. The original observation of a gender difference led to the formulation and confirmation of a survival hypothesis.

When CK5/6 has been tested on histological material from lung tumors, reactivity has been found in a small proportion of lung adenocarcinomas $(14,15)$. In the present study, the frequency of CK5/6 positivity was considerably higher than in reports based on the results obtained with histological sections.

The biological reasons for discrepancies between immunoreactivity in primary tumors and metastatic cells in effusions include the fact that the immunophenotype may change during tumor progression, and cells that are shed into effusions may reveal another immunophenotype than cells fixed in solid tissue (15-18).

Differences may also be due to the existence of aggressive subpopulations with high metastatic potential in the primary tumor. In an experimental study, Gemma et al found that highly metastatic subpopulations in a lung adenocarcinoma cell line had down-regulated CEA compared to the paternal cell line (19). Tumors causing malignant effusions have revealed their aggressive nature by spreading to the serous cavities. Therefore, it is reasonable to assume that the malignant cells found in the effusion reflect the antigen profile of such subpopulations.

The different ways to prepare and fix cytological and histological specimens for immunostains are numerous and makes comparison and standardization of immunoreactivity difficult (20). The method of preparation and fixation may affect the results, as shown by Shidham et al for the antikeratin antibody AE1/AE3 (21). The antigeneity of cytokeratins and other intraepithelial filaments is known to be diminished by formalin fixation, probably because cross-linking occurs between peptide molecules, masking the epitope sites.

The association of CK5/6 reactivity in effusions from NSCLC to longer survival has never been reported to our knowledge. If confirmed, the large difference in expected survival, weeks vs. months, would have obvious significance for the clinical management of the disease. All types of NSCLC are clinically equivalent in terms of treatment and prognosis, and prognostic factors are keenly sought (22). The finding also raises intriguing cancer biology issues about the mechanism connecting keratins to survival and the causes of gender difference.

Keratins are intermediate filaments present in the cytoplasm of epithelial cells, with different keratins being expressed in various cell types. Intermediate filaments form an elaborate network in the cytoplasm of most cells, providing a scaffold that integrates components of the cytoskeleton and organizes the internal structure of the cell.

The keratin filaments of epithelial cells are tightly anchored to the plasma membrane in areas of specialized contact areas, i.e. desmosomes and hemidesmosomes, between adjacent cells and between cells and underlying connective stroma. In the desmosomes, the cell-cell contacts are mediated by transmembrane proteins related to the cadherins $(10,23)$. There is evidence to suggest that altered expression of cadherins may be involved in invasion and metastasis of tumor cells. Cadherins are $\mathrm{Ca}^{2+}$-dependent cell-cell adhesion molecules, which interact with intracellular proteins called catenins. Alterations in the expression of the epithelial E-cadherin has been found in several carcinomas, including lung cancer, and cadherin is believed to play an important role in tumorgenicity and the metastatic process (24). Upregulation of E-cadherin is found in metastatic ovarian carcinoma cells in effusions, when compared with sections from the corresponding primary tumors, as reported by Davidson et al (25).

Research has revealed a correlation between the expression of cytokeratins in some malignant tumors and outcome. Thus, cytokeratin 6 expression in sections from primary gastric carcinomas was related to better clinical outcome (26).

In conclusion, we found a striking difference in survival times between patients with CK5/6-positive and -negative tumor cells in effusions. This is assumed to reflect a true biological difference. Whether this difference is also reflected in the immunophenotype of corresponding primary tumors or due to tumor progression or a difference between cells that are shed into fluid compared to those adherent to surrounding tissue cannot be elucidated from our results.

Lung cancer that has spread to the pleural cavity is consistent with an advanced stage tumor, and the prognosis is always dismal. However, CK5/6 reactivity in the cells found in the effusion predicts a considerably longer survival and may be of value for predicting the outcome in late stages of lung cancer. The difference in CK5/6 reactivity between men and women could explain the longer survival times found among men. The results indicate that keratins play a role in the aggressiveness of a subtype of NSCLC. 


\section{Acknowledgements}

Support from Malmö University Hospital Cancer Foundation is gratefully acknowledged.

\section{References}

1. Patel JD, Bach PB and Kris MG: Lung cancer in U.S. women a contemporary epidemic. JAMA 291: 1763-1768, 2004.

2. Olak J and Colson Y: Gender difference in lung cancer: have we really come a long way, baby? J Thor Cardiovasc Disease 128: 346-351, 2004.

3. Sugiura S, Ando, Y, Minami H, Ando M, Sakai S and Shimokata K: Prognostic value of pleural effusion in patients with non-small cell lung cancer. Clin Cancer Res 3: 47-50, 1997.

4. Mott FE, Sharma N and Ashley P: Malignant pleural effusion in non-small cell lung cancer-time for a stage revision? Chest 119 : 317-318, 2001.

5. Blobel GA, Moll R, Franke WW, Kayser KW and Gould VE: The intermediate filament cytoskeleton of malignant mesotheliomas and its diagnostic significance. Am J Pathol 121: 235-247, 1985

6. Moll R: Molecular diversity of cytokeratins: significance for cell and tumor differentiation. Acta Histochem Suppl 41: 117-127, 1991.

7. Clover J, Oates J and Edwards C: Anti-cytokeratin 5/6. a positive marker for epithelial mesothelioma. Histopathology 31 : 140-143, 1997.

8. Ordonez NG: Value of cytokeratin 5/6 immunostaining in distinguishing epithelial mesothelioma of the pleura from lung adenocarcinoma. Am J Surg Pathol 22: 1215-1221, 1998.

9. Ordonez NG: The immunohistochemical diagnosis of mesothelioma: a comparative study of epitheloid mesothelioma and lung adenocarcinoma. Am J Surg Pathol 27: 1031-1051, 2003.

10. Chu PG and Weiss LM: Keratin expression in human tissues and neoplasms. Histopathology 40: 403-439, 2002.

11. Fetsch PA and Abati A: Immunocytochemistry in effusion cytology. A contemporary review. Cancer (Cancer Cytopathol) 93: 293-308, 2001 .

12. Dejmek A and Hjerpe A: The combination of CEA, EMA and BerEp4, and hyaluronan analysis specifically identifies $79 \%$ of all histologically verified mesotheliomas causing an effusion. Diagn Cytopathol 32: 160-166, 2005.

13. Silverman J: Effusion cytology of metastatic malignancy of unknown primary. Pathol Case Rev 6: 154-160, 2001
14. Cury PM, Butcher DN, Fisher C, Corrin B and Nicholson AG: Value of the mesothelium-associated antibodies thrombomodulin, cytokeratin 5/6, calretinin, and $\mathrm{CD} 44 \mathrm{H}$ in distinguishing epithelioid pleural mesothelioma from adenocarcinoma metastatic to the pleura. Mod Pathol 13: 107-112, 2000.

15. Ramaekers FCS, Haag D, Kant A, Moesker O, Jap PH and Vooijs GP: Coexpression of keratin- and vimentin-type intermediate filaments in human metastatic carcinoma cells. Proc Natl Acad Sci USA 80: 2618-2622, 1983.

16. Berner HS, Davidson B, Berner A, et al: Expression of CD44 in effusions of patients diagnosed with serous ovarian carcinomadiagnostic and prognostic implications. Clin Exp Metastasis18: 197-202, 2000.

17. Davidson B, Risberg B, Goldberg I, et al: Ets-1 mRNA expression in effusions of serous ovarian carcinoma patients is a marker of poor outcome. Am J Surg Pathol 25: 1493-1500, 2001. (Erratum in: Am J Surg Pathol 26: 539, 2002).

18. Davidson B, Risberg B, Reich R and Berner A: Effusion cytology in ovarian cancer: new molecular methods as aids to diagnosis and prognosis. Clin Lab Med 23: 729-754, 2003.

19. Gemma A, Takenaka K, Hosoya Y, et al: Altered expression of several genes in highly metastatic subpopulations of a human pulmonary adenocarcinoma cell line. Eur J Cancer 37: 1554-1561, 2001.

20. Leong AS: Pitfalls in diagnostic immunohistology. Adv Anat Pathol 11: 86-93, 2004.

21. Shidham VB, Lindholm PF, Kajdacsy-Balla A, Chang CC and Komorowsky R: Methods of cytologic smear preparation and fixation. Effect on the immunoreactivity of commonly used anticytokeratin antibody AE1/AE3. Acta Cytol 44: 1016-1022, 2000 .

22. Watine J: Prognostic evaluation of primary non-small cell lung carcinoma patients using biological fluid variables. A systematic review. Scand J Clin Lab Invest 60: 259-273, 2000.

23. Cooper GM: The Cell - A Molecular Approach. 2nd edition. Sinauer Associates Inc., Sunderland, 2000.

24. Charalabopoulos K, Gogali A, Kostoula OK and Constantopoulos SH: Cadherin superfamily of adhesion molecules in primary lung cancer. Exp Oncol 26: 256-260, 2004.

25. Davidson B, Berner A, Nesland, et al: E-cadherin and alpha-, beta-, and gamma-catenin protein expression is up-regulated in ovarian carcinoma cells in serous effusions: J Pathol 192: 460-469, 2000.

26. Kim MA, Lee HS, Yang HK and Kim WH: Cytokeratin expression profile in gastric carcinomas. Hum Pathol 35: 576-581, 2004 . 